\title{
Assessing our ambulatory care time performance against the Royal College of Physicians time targets (Goal 5) and evaluating reasons for delay
}

\author{
Authors: Mark Hughes, ${ }^{\mathrm{A}}$ Eleonora Chakarova, ${ }^{\mathrm{B}}$ Anabella Dutiro, ${ }^{\mathrm{C}}$ Auditi Naziat ${ }^{\mathrm{D}}$ and Marian Chan $^{\mathrm{E}}$
}

\begin{abstract}
Aims
The purpose of this study is to assess whether patients attending the Ambulatory Care Centre (ACC) are seen within the target time of 4 hours and to compare against principle 5 of the ambulatory emergency care (AEC) toolkit of the Royal College of Physicians. ${ }^{1}$ We also looked to analyse the reasons for delay with those patients spending more than 4 hours in the department.
\end{abstract}

\section{Methods}

This was a retrospective snapshot of our services. Patients were identified using our data registration Excel sheets. Data were collected for a period of 1 week, from 27 March 2017 to 2 April 2017. The reception log book was reviewed to determine patient arrival and departure times. Our ICE (Integrated Clinical Environment) results reporting system was analysed to determine:

1 time bloods were taken

2 time bloods were reported

3 time radiology scan was performed and reported.

Patient notes were assessed to determine documented time of nurse and doctor review.

\section{Results}

Over the course of 1 week, 199 patients attended ACC; of those, 139 required doctor review. Timely nursing assessment (within 15 minutes) was achieved in $100 \%$ of patients. Of the 139 patients who required doctor review, review time was not recorded in 11 cases $(7.9 \%)$. Of the 128 patients for whom time of doctor review was recorded, 55 (43.0\%) were seen within 60 minutes of arrival. Initial doctor review was delayed by more than 1 hour for 73 patients $(57.0 \%)$.

\footnotetext{
Authors: ${ }^{A}$ Rheumatology, North West Anglia, Peterborough, UK; ${ }^{B}$ General Medicine, Luton and Dunstable Hospital, Luton, UK; ' Ambulatory Care, Luton and Dunstable Hospital, Luton,

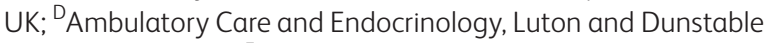
Hospital, Luton, UK; ${ }^{\mathrm{E}}$ Rheumatology, Luton and Dunstable Hospital, Luton, UK
}

The commonest reason for delay in medical review was time spent waiting for blood results to be reported in 20 out of 66 cases $(30 \%)$, with an average time for bloods being received in the laboratory to being reported of 148 minutes. The second most common reason was delay in radiology reporting, in 13 of the 66 cases $(19.7 \%)$. The third most common delay was for five of 66 patients (7.6\%) who were awaiting medical admission.

The average wait for computed tomography (CT) or ventilation/ perfusion scans was 124 minutes. None of the five patients who attended ACC for a magnetic resonance imaging (MRI) scan spent more than 4 hours in the department, probably due to the fast-track MRI scanning slots reserved for ACC patients.

Waiting time for blood results at the weekend was 85 minutes, slightly better than the overall average of 103 minutes, indicating that the current service is functioning well at weekends.

\section{Conclusions}

Understanding the reasons for delay in medical review is important in order to align our services to meet time targets.

> We are able to demonstrate to both our laboratory and radiology services the need for rapid reporting for ACC.

> Furthermore, we are also able to highlight the need for ACC admissions to be prioritised, particularly when we have fixed opening hours.

> We also need to change the culture of waiting for reports before seeing patients, and using telephoning or results letters instead. This will enable swifter medical assessment from arrival.

> Potentially, a patient flow-tracking system similar to that used in the emergency department will allow us to monitor these targets and parameters in real time, allowing for more responsive solutions.

\section{Conflict of interest statement}

No conflicts of interest for any of the authors to declare.

\section{Reference}

1 Royal College of Physicians. Acute care toolkit 10: Ambulatory emergency care. London: RCP, 2014. www.rcplondon.ac.uk/act10 [Accessed 24 January 2019]. 